\title{
Peak Detection Algorithm for Laser Induced Breakdown Spectroscopy
}

\author{
Bo Zhang ${ }^{1,2}$, Haibin $\mathrm{Yu}^{1}$, Lanxiang Sun ${ }^{1}$ \\ ${ }^{1}$ Lab. of Networked Control Systems, Shenyang Institute of Automation, Chinese Academy of Sciences \\ ${ }^{2}$ University of Chinese Academy of Sciences, Beijing 100039, PR China \\ e-mail: zhangbo@sia.cn (B.Zhang),yhb@sia.cn(H.Yu), sunlanxiang@sia.cn (L.Sun)
}

\begin{abstract}
Peak detection is an essential step in LIBS data analysis. We present a peak detection method, which is based on continuous wavelet transform. We compare three commonly methods of peak detection and discuss their performance.
\end{abstract}

Keywords-peak detection; Laser induced breakdown spectroscopy; continuous wavelet transform

\section{INTRODUCTION}

Laser-induced breakdown spectroscopy (LIBS) is a type of atomic emission spectroscopy. It can analyse the matter with any physical state and without any sample preparation [1-4]. LIBS transfers the physicochemical property to measurable peak on a certain wavelength. Peak is viewed as a signal which has larger amplitude than that of proximity region. Several methods are used to solve peak detection. Some methods are suitable for a certain type of signal and have reliability performance, but they have poor performance on other type of signal. Therefore the peak detection method is appropriate for the specific character of LIBS signal.

In general, the peak detection methods can fall into three types. The first type is based on the signal to noise ratio (SNR) [5]. This is very simple and effective peak detection method. The key step of this method is the noise definition. Noise is often defined by the median absolute deviation with different samples. After defining the noise we calculated the signal to noise ratio. Peaks are identified by finding several local maximum with a larger SNR than a threshold. The second type of peak detection method is searching peak shape like a certain model function, such as Gaussian function [6]. With a small running window, the method detects LIBS signals and calculates the correlation coefficient with Gaussian function. Thus the LIBS signals are turned into a vector of correlation coefficients. If the correlation coefficients are higher than a certain threshold, then the corresponding signals are defined as peaks. The third type is the case that based on continuous wavelet transform (CWT) [7]. The key part of this method is using CWT under a certain level for peak detection. However, it is difficult to choose the proper CWT levels for different spectrum. In a word, the methods described above have different intentions of peak detection.

In this paper we present a peak detection method for LIBS signals based on CWT. Furthermore, we have compared three different peak detection methods (SNR, Template-based approach and CWT) with different performance.

\section{ALGORITHMS}

There are several evaluation indexes of peak detection, such as SNR and local maximum. Firstly, a brief introduction to continuous wavelet transformation (CWT) is presented. The process of CWT is represented as follows:

$$
W(a, b)=\int_{-\infty}^{+\infty} f(t) \psi_{a, b}(t) d t=\frac{1}{\sqrt{a}} \psi\left(\frac{t-b}{a}\right), a \in R^{+}, b \in R(1)
$$

where $f(t)$ is the signal, $a$ is called scale parameter, $b$ is called translation parameter, and $\Psi$ is called mother wavelet function. The mother wavelet should have the basic characteristics of a peak signal, because the similarity between the signal $f$ and $\Psi$ are supposed to embody by the wavelet coefficients [8]. The Mexican Hat wavelet was used as the mother wavelet, which can be described below:

$$
\psi(x)=\left(\frac{2}{\sqrt{3}} \pi^{-1 / 4}\right)\left(1-x^{2}\right) e^{-x^{2} / 2}
$$

When the CWT is applied to LIBS data, some local maximum corresponding to the peak can be found in the wavelet coefficients under different levels. In a certain position, the wavelet coefficients under each level are all have local maximum, so the peak exists on this position. The ridges are obtained by these local maximum of the wavelet coefficients under each level. In addition, three constraint criterions are used to obtain the ridges: (1) the scale of the local maximum on the ridges; (2) SNR should higher than a threshold; (3) the length of the ridge should higher than a threshold. Based on the ridges, the peak detection method for LIBS signal have three key steps: (1) drawing the ridges by linking the local maxima; (2) identifying the peaks based on the ridges lines with three constraint criterions; and (3) detection peaks of the LIBS signal.

\section{SIMULATION AND EXPERIMENT}

To demonstrate the feasibility of the proposed method for searching peaks, simulated data with the known peaks has been performed. On the other hand, LIBS experimental setup is prepared to test and verify the proposed method in practical applications. For obtaining spectral data in this work, this LIBS experimental setup is shown in Fig. 1. The laser source is a Q-switched Nd:YAG laser with maximum pulse energy of $200 \mathrm{~mJ}$, pulse width of 12-15ns, wavelength of $1064 \mathrm{~nm}$, and repetition rate of $1-15 \mathrm{~Hz}$. The sample is placed on a three-dimensional manually controlled stage, and the laser beam is focused on the sample surface with a convergent lens of $75 \mathrm{~mm}$ focal length. The spectrometer is ESA 4000 
from LLA Instruments $\mathrm{GmbH}$ for analysis. In a wavelength range of $200 \mathrm{~nm}$ to $780 \mathrm{~nm}$ and with a resolution of a few picometers spectra are recorded by an ICCD camera. The samples used in experiment are some national standard copper alloy samples, and their serial number is GSB 04-2416-2008. In the measurement, the laser pulse energy is $100 \mathrm{~mJ}$, the laser fluence on sample is $50 \mathrm{~J} / \mathrm{cm} 2$, and the single laser pulse frequency is $5 \mathrm{~Hz}$. Because LIBS data are influenced by a variety of experimental factors in the test process, the repeatability is low. For this reason, we use the average of multi-measurement to reduce the error caused by the uneven distribution of inner components. We measured each sample for 10 times in five different positions. All experiments are under the conditions of normal atmospheric pressure and 25 degree centigrade.

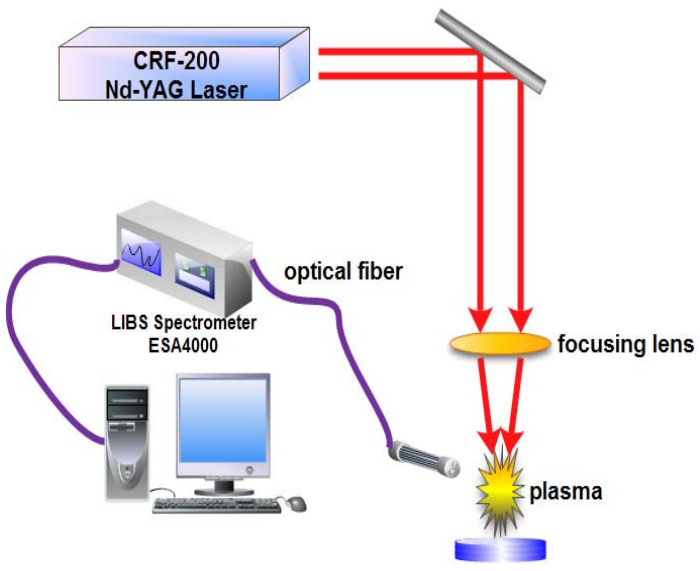

Figure 1.LIBS Schematic visualization of the set-up for LIBS experiment.

\section{RESUlTS AND Discussion}

To verify the validity of the proposed method, the simulated LIBS signal is illustrated here. The CWT was performed for scales from 1 to 32. The wavelet coefficients are presented as 2D image, in which the horizontal axis corresponds to the wavelength and the vertical axis to the scales. The amplitude values of the coefficients are shown in a grey scale (Fig. 2(a)). From Fig. 2(b) and Fig. 2(c), the ridges have the same positions corresponding to the peaks.
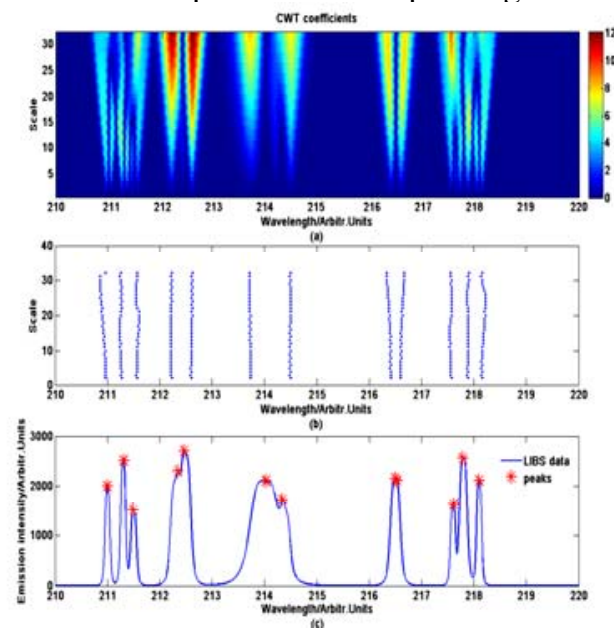

Figure 2. Peak detection method. (a) Grey scale image of the 2D wavelet coefficients (red means high and blue means low). (b) The ridges lines obtained by the 2D wavelet coefficients. (c) The simulated LIBS signal.
In this paper, we carry out a comparison of the proposed method with other peak detection methods. In Fig. 3, three different methods show different sensitivity to peak detection, specifically, SNR method and template-based method ignore some peaks obviously.

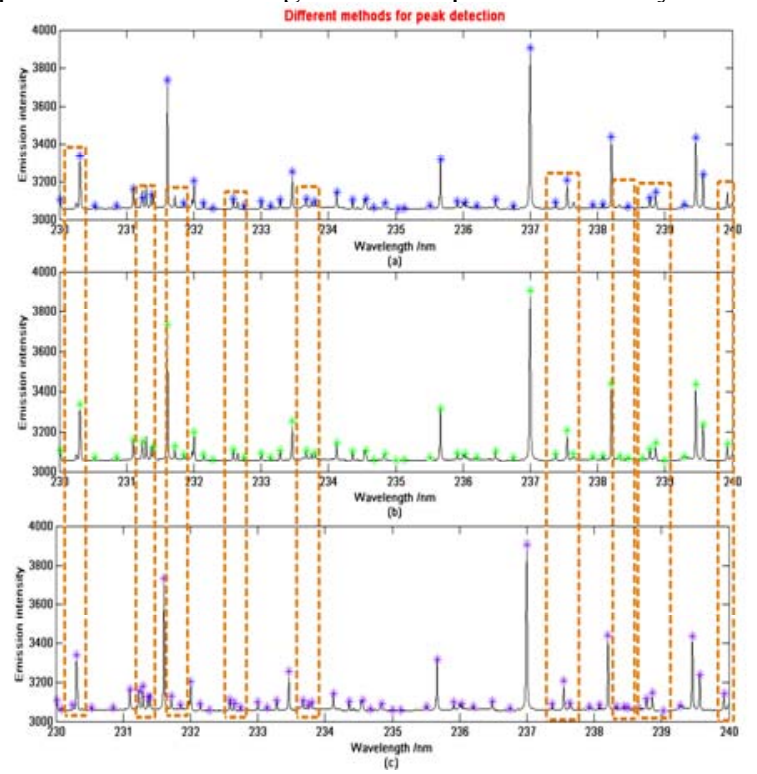

Figure 3.Different methods for peak detection in LIBS. (a) SNR method. (b) template-based method. (c) CWT.

The features of SNR method are fast computation, ease of implement and good smoothing performance. But this method ignores the shape of the peak, so the wrong judgment is high. In addition, the noise is an essential part of this method and therefore strongly depends on SNR of the signal. Based on the assumption of the peak shape like Gaussian function, the template-based method depends on the experimental parameters, particularly laser energy and delay time, which lead total the significant change of peak model. This method has robust for lower SNR, but the high noise level may result in the poor performance of peak detection. Unlike SNR method, the CWT method is complex but is very robust even for lower SNR. Since wavelet transform has the ability to decompose signal into the wavelet coefficients, the wavelet coefficients of the noise are smaller than that of signal. When searching the ridges, the smaller wavelet coefficients are removed.

\section{CONCLUSIONS}

In this paper, CWT method is applied to detect peaks for LIBS. SNR method is fast computation and ease of implement but sensitive to SNR. Template-based method is better than SNR method, but the performance of this method depends on the template function, which is like peak model. CWT method shows better performances than other methods, and this method is stable even for lower SNR. Therefore, for improved peak detection accuracy, CWT method is suggested.

\section{ACKNOWLEDGMENT}

This work has been supported by National Natural Science Foundation of China (Grant No.61004131) and the National High-Tech Research and Development Program of China (Grant No.2012AA040608). The authors would like to thank LIBS project team of SIA (Shenyang Institute 
of Automation, Chinese Academy of Sciences) for profitable recommendations for improving method.

\section{REFERENCES}

[1] Richard A Myers, Noah J Kolodziejski, Michael R Squillante. "Commercialization of laser-induced breakdown spectrscopy for lead-in-paint inspection,” Appl. Opt. vol.47, NO.31, pp. 7-14, August 2008

[2] Valeri I Babushok, Frank C DeLucia. "Kinetic modeling of the laser-induced break- down spectroscopy plume from metallic lead,” Appl.Opt.vol.42,NO.30,pp.5497-5512, September 2003

[3] J. Diedrich, S.J. Rehse, S. Palchaudhuri. "Escherichia coli identification and strain discrimination using nanosecond laser-induced breakdown spectroscopy,” Appl. Phys. Lett. Vol.90, pp.163901-163903, January 2007.

[4] A. Ferrero and J. J. Laserna. "A theoretical study of atmospheric propagation of laser and return light for stand-off laser induced breakdown spectroscopy purposes,” Spectrochim. Acta Part B vol.63, pp.305-311, June 2008.

[5] C. Yang, Z. He, X. Wan, Q. Yang, H. Xue and W. Yu. "A Filtering-based Approach for Detecting Epistatic Interactions in Genome-wide Association Studies,” Bioinformatics, vol.25,pp. 504-511, 2009.

[6] R.Redon,B.Fermat,J.Richou. "A reconvolution spectroscopic method,” J.Quant.Spectrosc.Radiat.Transf.vol 58,pp 151-170,1997.

[7] K.R. Coombes, S. Tsavachidis, J.S. Morris, K.A. Baggerly, M.C. Hung, and H.M. Kuerer. "Improved peak detection and quantification of mass spectrome-try data acquired from surface-enhanced laser desorption and ionization by de-noising spectra with the undecimated discrete wavelet transform". Proteomics, vol.5, pp.4107-4117, November 2005.

[8] Du P, Kibbe WA, Lin SM. "Improved peak detection in mass spectrum by incorporating continuous wavelet transform-based pattern matching,” Bioinformatics,vol.22,pp.2059-2065, July 2006. 